\title{
Cardiac CZT-SPECT: More than left ventricular imaging
}

\author{
Bo Zerahn, MD, a and Christian Haarmark, MD PhD ${ }^{\mathrm{a}, \mathrm{b}}$ \\ a Department of Nuclear Medicine, Copenhagen University Hospital - Herlev and Gentofte, \\ Copenhagen, Denmark \\ b Department of Clinical Medicine, University of Copenhagen, Copenhagen, Denmark
}

Received Jun 8, 2021; accepted Jun 8, 2021

doi: $10.1007 / \mathrm{s} 12350-021-02701-y$

\section{See related article, pp. 1647-1656}

The concept of equilibrium radionuclide nuclear angiography (ERNA) has come a long way since it was first introduced in the late 1970s as the gated equilibrium blood-pool time activity method where left ventricular ejection fraction (LVEF) could be determined from planar images and soon after also right ventricular ejection fraction (RVEF) from first pass imaging ${ }^{1,2}$ being a new alternative to cardiac catheterization with contrast ventriculography. Non-invasive and radiation free methods for assessment of cardiac function has since then emerged in the shape of MRI, bioelectric impedance, indicator dilution technique and echocardiography along with further development of radioactive tracer techniques such as myocardial perfusion imaging and cardiac CT as a radiographic alternative. This multitude of available methods for determination of cardiac function has inevitably increased the demand for comparative studies in order to evaluate which methods best serve a given patient population in terms of information provided along with accuracy, reproducibility, availability, cost, risk and practicability. One of the emerging modalities is Cadmium Zinc Telluride gated SPECT equilibrium radionuclide angiography (CZTERNA). ${ }^{3,4}$

In this context the study in this issue of Journal of Nuclear Cardiology by Apert et al. ${ }^{5}$ fills in a part of the gap of lacking information on this subject with their

Reprint requests: Christian Haarmark, MD PhD, Department of Clinical Medicine, University of Copenhagen, Copenhagen, Denmark; haarmark@dadlnet.dk

J Nucl Cardiol 2022;29:1657-9.

$1071-3581 / \$ 34.00$

Copyright (C) 2021 American Society of Nuclear Cardiology. examination of right ventricular volumes and ejection fraction in patients with cardiomyopathy. Apert and colleagues are among the first to report on the reproducibility and accuracy of CZT-ERNA compared to cardiac magnetic resonance imaging (CMR) in patients with cardiomyopathy. They examined twenty-one patients with cardiomyopathy (both ischemic and nonischemic) who were referred for the evaluation of possible later implantation of left ventricular assist devices. The right ventricle is of importance in this setting, as it is left non-assisted and right ventricular failure can be seen post implantation. They found that CZT-ERNA provides accurate and reproducible results regarding the right ventricle when compared to CMR even in a population with reduced LVEF_-yielding yet a promising indication for cardiac CZT scans. Especially the fact that the right ventricular volumes are comparable to those obtained from CMR, is important as right ventricular function is more difficult to assess due to its thin myocardium, irregular shape and difficulties defining the valvular plane of the pulmonary artery as compared to the left ventricle.

There are limitations specifically related to the CZT technique itself, also denoted in the study by Apert and colleagues. A total of 7 patients could not be evaluated either due to irregular heart rhythm (3 patients) or due to centering problems which made RV evaluation impossible (4 patients). Irregular heart rhythm is a potential confounder for most non-invasive techniques when evaluating right or left ventricular function-including CMR. Nonetheless, ERNA recorded in list mode enables re-binning of the acquisition for analysis of user selected $\mathrm{R}-\mathrm{R}$ intervals in cases with irregular heart rhythm, ${ }^{6,7}$ which is not available for CMR and echocardiography to the same extent. With the CZT camera at hand for Apert et al. there is an automated function for "finding the heart' prior to acquisition, which works for myocardial perfusion imaging only. When it comes to ERNA this is 
an operator-dependent procedure potentially reducing practicability of ERNA.

Upon introduction of new techniques, the practicability of the method is always of importance-thus for the current ERNA technique to be generally accepted and taken up for general use- a four out of twenty-five patients $=16 \%$ failure rate due to centering problems is perhaps too high in a clinical setting. However, in a far larger study with routine monitoring of LVEF with CZT-ERNA the technique has proven practicable in 98\% of referrals. Of the remaining $2 \%$ of referrals ERNA could be achieved with either planar or Natrium Iodide (NaI)-SPECT in the majority of cases. Only $0.5 \%$ of all referrals required estimation of cardiac function with other modalities. ${ }^{8}$ Most cases where CZT-ERNA was not possible were due the inability of some patients to raise their left arm above their head (1\%) and large thoracic circumference $(0.5 \%)$. Another drawback with the study by Apert et al. is the relative low number of included patients, which does not allow for stratification into bins of near-normal to very-low RVEF, to test whether the performance of CZT-ERNA compared to CMR is stable at very-low RVEF as well. Nonetheless, the results are still quite convincing for the patient group as a whole.

CZT cameras for ERNA also have other advantages than measuring right ventricular function compared to traditional gamma cameras. It is possible to reduce both examination time ${ }^{9}$ - thus increasing throughput and availability - and reduce administered tracer dose especially if using individually adapted dosage. ${ }^{10}$ The latter is particularly important for patients requiring continuous cardiac monitoring while undergoing potentially cardiotoxic treatment. The same holds true for patients in the need for cardiac function monitoring for other indications where echocardiography or CMR are either unavailable or unsuitable. Moreover the reduced patient dose will inevitably lead to reduced dose to the staff and even reduced cost as both tracer and radioisotope consumption decline.

Another feature with the CZT-ERNA technique that has been demonstrated by others and once again confirmed by Apert et al in the study in this issue is the low variability of cardiac measurements. ${ }^{11}$ This high reproducibility extends to other variables than RVEF/LVEF such as peak emptying and filling rates (PER and PFR, respectively) for both left and right ventricles as well as time to peak filling rate (TTPFR). Moreover reference ERNA data for CZT-SPECT in chemotherapy naïve cancer patients have been published for both LVEF and RVEF along with ventricular volumes, PER, PFR and TTPFR. ${ }^{4,12}$ Thus the diagnostic certainty is increased when evaluating cardiac variables and changes during a course of potentially cardiotoxic treatment or other interventions. Apert et al also indicated, that manual intervention were more often required and took longer for CMR images than for CZT studies, were the automated QBS software was a clear advantage for the latter. From a research point of view, a study using tests with such practicability, reproducibility and low variability will inevitably require fewer included patients than studies using tests with poorer performance and subsequently shorter study duration and lower costs. Nonetheless, it is still mandatory to evaluate in detail for each cardiotoxic regime or other monitoring indication to what degree the better practicability and other qualities of CZT-ERNA outweighs the potential risks of using a radioactive tracer for routine continuous monitoring of cardiac function.

Even though CZT-SPECT cameras are as yet considerably more expensive than their $\mathrm{NaI}$ counterparts they do provide improved imaging quality, faster acquisition and lower radioactive dose to both patients and staff. Moreover, they provide a practicability beyond what can be achieved with both MRI and echocardiography for both myocardial perfusion imaging and ERNA although backup NaI gamma camera systems may still be required for some patients.

All in all, the study by Apert and colleagues add to the cumulating evidence that CZT-ERNA is a reliable method for the evaluation of both right and left ventricular volumes and function-also in the setting of cardiomyopathy. This further underlines the many facets and possible indications for cardiac CZT-SPECT, not only for RVEF/LVEF assessment using ERNA SPECT, but also for other well-established indications such as myocardial perfusion imaging ${ }^{13}$ and newer not yet fully established indications such as amyloidosis ${ }^{14}$ and the possibility for dual isotope studies, i.e., combined WBC scan/perfusion studies. ${ }^{15}$

\section{Declarations}

\section{Disclosure}

The authors have no conflict of interest to declare.

\section{References}

1. Steele P, Kirch D, LeFree M, Battock D. Measurement of right and left ventricular ejection fractions by radionuclide angiocardiography in coronary artery disease. Chest 1976;70:51-6. https://doi. org/10.1378/chest.70.1.51

2. Maddahi J, Berman DS, Matsuoka DT, et al. A new technique for assessing right ventricular ejection fraction using rapid multiplegated equilibrium cardiac blood pool scintigraphy. Description, validation and findings in chronic coronary artery disease. Circulation 1979;60:581-89. https://doi.org/10.1161/01.CIR.60.3.581 
3. Jensen MM, Schmidt U, Huang C, Zerahn B. Gated tomographic radionuclide angiography using cadmium-zinc-telluride detector gamma camera; comparison to traditional gamma cameras. J Nucl Cardiol 2014;21:384-96.

4. Haarmark C, Haase C, Jensen MM, Zerahn B. Pre-chemotherapy values for left and right ventricular volumes and ejection fraction by gated tomographic radionuclide angiography using a cadmiumzinc-telluride detector gamma camera. J Nucl Cardiol 2016;23:8797. https://doi.org/10.1007/s12350-015-0177-5

5. Apert A, Canu M, Jankowski A, et al. Comparison of Cadmium Zinc Telluride ECG-gated SPECT equilibrium radionuclide angiocardiography to magnetic resonance imaging to measure right ventricular volumes and ejection fraction in patients with cardiomyopathy. J Nucl Cardiol 2021. https://doi.org/10.1007/s1 2350-021-02653-3

6. Kim BH, Ishida Y, Tsuneoka Y, et al. Effects of spontaneous respiration on right and left ventricular function: Evaluation by respiratory and ECG gated radionuclide ventriculography. J Nucl Med 1987;28:173-77.

7. DePuey EG, Almasi JJ, Eisner RL. Advantages of list-mode data acquisition in gated blood-pool studies. Texas Hear Inst J 1984;11:268-74.

8. Rydberg J, Andersen J, Haarmark C, Zerahn B. The influence of anthropometric and basic circulatory variables on count rate in cadmium-zinc-telluride SPECT gated radionuclide angiography. J Nucl Cardiol 2019. https://doi.org/10.1007/s12350-018-1402-9

9. Herzog BA, Buechel RR, Katz R, et al. Nuclear myocardial perfusion imaging with a cadmium-zinc-telluride detector technique: Optimized protocol for scan time reduction. J Nucl Med 2010;51:46-51. https://doi.org/10.2967/jnumed.109.065532
10. Duvall WL, Guma-Demers KA, George T, Henzlova MJ. Radiation reduction and faster acquisition times with SPECT gated blood pool scans using a high-efficiency cardiac SPECT camera. J Nucl Cardiol 2016;23:1128-38. https://doi.org/10.1007/s12350-0 15-0214-4

11. Romero-Farina G, Aguadé-Bruix S. Equilibrium radionuclide angiography: Present and future. J Nucl Cardiol 2019. https://doi. org/10.1007/s12350-019-01876-9

12. Hansen NL, Haarmark C, Zerahn B. Ventricular peak emptying and filling rates measured by gated tomographic radionuclide angiography using a cadmium-zinc-telluride SPECT camera in chemotherapy-naïve cancer patients. J Nucl Cardiol 2020;27:1193-1201. https://doi.org/10.1007/s12350-019-01756-2

13. Duvall WL, Sweeny JM, Croft LB, et al. Comparison of high efficiency CZT SPECT MPI to coronary angiography. J Nucl Cardiol 2011;18:595-604. https://doi.org/10.1007/s12350-011-93 $82-\mathrm{z}$

14. Flaherty KR, Morgenstern R, Pozniakoff T, et al. 99mTechnetium pyrophosphate scintigraphy with cadmium zinc telluride cameras is a highly sensitive and specific imaging modality to diagnose transthyretin cardiac amyloidosis. J Nucl Cardiol 2020;27:371-80. https://doi.org/10.1007/s12350-019-01831-8

15. Caobelli F, Wollenweber T, Bavendiek U, et al. Simultaneous dual-isotope solid-state detector SPECT for improved tracking of white blood cells in suspected endocarditis. Eur Heart $\mathbf{J}$ 2017;38:436-43. https://doi.org/10.1093/eurheartj/ehw231

Publisher's Note Springer Nature remains neutral with regard to jurisdictional claims in published maps and institutional affiliations. 